\title{
Proteome comparisons reveal influence of different dietary proteins on the development of rat jejunum
}

\author{
Mengjie LI ${ }^{1}$, Chunbao LI ${ }^{1}$, Shangxin SONG ${ }^{2}$, Xinglian XU ${ }^{1}$, Guanghong ZHOU (凶) ${ }^{1}$ \\ 1 Key Laboratory of Meat Processing and Quality Control/Key Laboratory of Meat Processing; Jiangsu Collaborative Innovation Center of \\ Meat Production, Processing and Quality Control, Nanjing Agricultural University, Nanjing 210095, China \\ 2 School of Food Science, Nanjing Xiaozhuang University, Nanjing 211171, China
}

\begin{abstract}
This study compared proteome profiles and morphological changes of rat jejunum in response to different dietary proteins. Fifty male Sprague-Dawley rats were fed with casein (control), and isolated beef, pork, fish and chicken proteins for 14 days. Proteome analysis, histological observation and PEPT1 quantification of the jejunum were performed. The results indicated that rats fed with chicken proteins had higher PEPT1 mRNA and protein levels $(P<0.05)$ but lower villus height and ratio of villus height to crypt depth $(\mathrm{V} / \mathrm{C}$ ratio, $P<0.05)$ than those fed with casein and pork protein. Label-free LC-MS/ MS indicated that, as compared to casein, intake of chicken protein can regulate oligopeptide transport mainly by upregulating PEPT1 protein expression and reducing dipeptidyl-peptidase activity related to biological oxidation, and can reduce oligopeptide absorption capacity by regulating Hippo signaling pathway. Although intake of beef and fish proteins had no significant effect on PEPT1 expression, they altered several signaling pathways.
\end{abstract}

Keywords Hippo signaling pathway, meat protein, PEPT1, proteome analysis, rat jejunum

\section{Introduction}

Dietary protein affects the intestinal morphology and amino acid transport. Previous studies indicated that a high protein diet increased amino acid uptake in the jejunum, while a protein-deficient diet reduced uptake of nonessential amino acids but increased uptake of essential amino acids and alanine ${ }^{[1]}$. Kuhla et al. ${ }^{[2]}$ observed that partial substitution of milk protein for soy protein increased the $\mathrm{V} / \mathrm{C}$ ratio of goat jejunum and changed the proteome profile of jejunum involving protein turnover,

Received June 19, 2017; accepted November 27, 2017

Correspondence: ghzhou@njau.edu.cn energy metabolism and cytoskeleton assembly, and those authors attributed the differences to the composition of essential amino acids. Numerous studies have focused on amino acids nutrition in the field of farm animal science $^{[3-6]}$. For example, glutamine supplementation can increase enterocyte proliferation ${ }^{[3]}$, but arginine supplementation may cause lower cell proliferation and greater enterocyte apoptosis ${ }^{[4]}$. Tryptophan and L-leucine were also shown to have a significant effect on intestinal morphology and tight junction proteins ${ }^{[5,6]}$.

Dietary proteins are digested and absorbed in the gastrointestinal tract. Peptide transporter PEPT1/ SLC15A1 plays a critical role in uptake of dipeptides and tripeptides from the lumen to the cells ${ }^{[7]}$. PEPT1 belongs to proton-coupled oligopeptide transporter superfamily, and is mainly located in the brush-border membrane of enterocytes ${ }^{[8,9]}$, and villus height and crypt depth of enterocytes reflect the gut absorption capacity ${ }^{[10,11]}$. Nutrient absorption is related to many aspects, in particular to the abundance of transmembrane transporters, energy metabolic enzymes and regulatory factors ${ }^{[12]}$. PEPT1 expression may be in turn regulated by the level and origin of dietary proteins ${ }^{[13-15]}$.

Nutritional values differ between plant and meat proteins ${ }^{[16,17]}$, because of different protein composition (especially the essential amino acid profiles) and digestibility ${ }^{[18]}$. Given that oligopeptide transporters play a more critical role in the uptake of digested proteins than amino acid transporters ${ }^{[19]}$, different protein sources might be expected to induce changes in PEPT1 expression. A previous study examined the effects of soy protein and four meat proteins on morphology and PEPT1 expression of rat duodenum and jejunum after 7 days feeding, and showed that beef, pork, chicken and fish protein in diets potentially have different effects on animal gut development ${ }^{[20]}$. However, few data or information about underlying mechanisms are available about the differences between these dietary proteins and implications for long-term feeding. 
This study aims to compare the differences in PEPT1, morphology, and proteome profiles in rat jejunum after feeding with casein (control), beef, pork, chicken and fish proteins for 14 days. Bioinformatics analysis revealed information about the possible underlying mechanism.

\section{Materials and methods}

\subsection{Diets and sampling}

\subsubsection{Diets}

Diets for growing rats were prepared according to the AIN$93 \mathrm{G}$ diet formulation ${ }^{[21]}$, with replacement of casein by the same quantity of isolated proteins from beef, pork, chicken or fish according to the treatment. Casein extract was sourced from Jiangsu Xietong, Inc. (Nanjing, China). Meat proteins were extracted as previously described ${ }^{[22]}$ with minor modifications. Briefly, pork and beef longissimus dorsi muscles, chicken pectoralis major muscle and fish (carp) dorsal muscle were cut, packed and cooked in a water bath at $70^{\circ} \mathrm{C}$. Then the cooked samples were ground and fats removed twice by methylene chloride/methanol $(2: 1, \mathrm{v}: \mathrm{v})$. The residual solvent in the powder was fully evaporated in a fume hood for $24 \mathrm{~h}$. The meat protein powders were sieved through a 25 mesh and the resulting powder contained greater than $92 \%$ protein and 6\%-8\% water. Diets were prepared by Jiangsu Xietong, Inc. (Nanjing, China). Details of these diets, including ingredient composition, have been reported by Song et al. ${ }^{[23]}$. The amino acid profiles in the diets are shown in the Table S1.

\subsubsection{Animals and sampling}

The animal experimental protocol was approved by the Ethics Committee of the Experimental Animal Center of Nanjing Agricultural University (license number SCXK (Su) 2002-0029). Fifty male Sprague-Dawley rats (3 weeks old) were obtained from Shanghai Laboratory Animal Research Center (Shanghai, China). Animals were housed in pairs at $23 \pm 2^{\circ} \mathrm{C}$ and with a $12 \mathrm{~h} \mathrm{light/dark} \mathrm{cycle.}$ To adapt to new conditions, all rats received the AIN-93G diet that contains casein for 7 days. After that, animals were divided into five diet groups (10 rats per group), i.e.,
AIN-93G (casein), beef protein, pork protein, chicken protein and fish protein. The animals were provided with diets and water ad libitum. After 14 days feeding (animal age: 42 days old), the rats were killed by cervical dislocation after $4 \mathrm{~h}$ deprival of diets. The jejunum was taken out and immediately rinsed with $0.9 \% \mathrm{NaCl}$ solution. Segments $(1 \mathrm{~cm})$ of the proximal jejunum were collected and fixed in $10 \%$ formalin buffer for $12 \mathrm{~h}$ for histological observations. The remainder of the jejunum was used for qRT-PCR, western blotting and proteomic analyses.

\subsection{Quantification of PEPT1}

\subsection{1 qRT-PCR}

The TaKaRa MiniBEST Universal RNA Extraction Kit (TaKaRa, Ostu, Japan) was used for total RNA extraction of jejunal samples according to the manufacturer's instructions. Total RNA was quantified by a NanoDrop ND-2000 spectrophotometer (NanoDrop Technologies, DE, USA). The RNA was reverse transcribed to cDNA using PrimeScript ${ }^{\mathrm{TM}}$ RT Master Mix (TaKaRa, Ostu, Japan) in a Peltier Thermal Cycler 200 (MJ Research, Watertown, MA, USA). The cDNA was dissolved in RNase-free water and stored at $-20^{\circ} \mathrm{C}$. The two-step qRTPCR reactions were performed in triplicate on 96-well plates using a 7500 Real-time PCR system (Applied Biosystems, Foster, CA, CA) with the SYBR ${ }^{\circledR}$ Premix Ex $\mathrm{Taq}^{\mathrm{TM}}$ (TaKaRa, Ostu, Japan). The Pept1 primers were designed using the Primer 3.0 (Applied Biosystems), and the Gapdh primers (reference gene) were provided by Sangon Biotech (Shanghai, China) (Table 1). The concentrations of template, primers and amplified products were evaluated by relative standard curves made by multiple dilution (1:1 to $1: 625)$. The reaction solution $(20 \mu \mathrm{L})$ contained $10 \mu \mathrm{L}$ SYBR ${ }^{\circledR}$ Premix Ex Taq, $0.4 \mu \mathrm{L}$ PCR forward primer $\left(10 \mu \mathrm{mol} \cdot \mathrm{L}^{-1}\right), 0.4 \mu \mathrm{L}$ PCR reverse primer $\left(10 \mu \mathrm{mol} \cdot \mathrm{L}^{-1}\right), 0.4 \mu \mathrm{L}$ ROX reference dye II, $2 \mu \mathrm{L}$ cDNA and $6.8 \mu \mathrm{L} \mathrm{dH}_{2} \mathrm{O}$. Cycling conditions were as follows: $30 \mathrm{~s}$ for denaturation at $95^{\circ} \mathrm{C}, 40$ cycles of $5 \mathrm{~s}$ at $95^{\circ} \mathrm{C}$ and $34 \mathrm{~s}$ at $60^{\circ} \mathrm{C}$ for denaturation, followed by three alternations between $95^{\circ} \mathrm{C}$ and $60^{\circ} \mathrm{C}$ for melting curve analysis to verify the specificity of a single amplification. Fold changes of Pept1 expression were calculated by the $2^{-\Delta \Delta C_{\mathrm{T}}}$ method $^{[24]}$ normalized to Gapdh, and casein group was set as the control.

Table 1 Primers used for qRT-PCR

\begin{tabular}{lcccccc}
\hline Gene & Primer & Sequence $\left(5^{\prime}-3^{\prime}\right)$ & Length/bp & Product size/bp & Tm/ ${ }^{\circ} \mathrm{C}$ & GenBank Accession no. \\
\hline Pept1 & Forward & GTA TGT TCT GTT CGC CTC CTT G & 22 & 228 & 60.07 & NC_005114.3 \\
& Reverse & GGT GAA TGC TGG ACT TGG TAT G & 22 & & 60.07 & \\
\multirow{2}{*}{ Gapdh } & Forward & ACA GCA ACA GGG TGG TGG AC & 20 & 252 & 57.70 & NC_005103.4 \\
& Reverse & TTT GAG GGT GCA GCG AAC TT & 20 & & 57.80 & \\
\hline
\end{tabular}




\subsubsection{Western blotting}

The mucosa was separated from jejunal samples and proteins were isolated from epithelial cells with a commercial protein extraction kit (Beyotime, Nantong, China) according to the manufacturer's instructions. To avoid any enzymatic reactions during preparation, two enzyme inhibitors (Sigma-Aldrich, St. Louis, MO, USA), i.e., protease inhibitor complete (one tablet per $50 \mathrm{~mL}$ ) and phosphatase inhibitor phosStop (two tablets per $50 \mathrm{~mL}$ ) were added into cold protein extraction buffers. Protein concentration was determined by a BCA protein assay kit (Pierce, Rockford, IL, USA). The protein extracts were lysed in $4 \times$ protein loading buffer (Solarbio, Beijing, China) and heated for $5 \mathrm{~min}$ at $80^{\circ} \mathrm{C}$. Proteins $(30 \mu \mathrm{g})$ were separated on $10 \%$ SDS polyacrylamide gels (Bio-Rad, Laboratories, Hercules, CA, USA) electrophoresed at $100 \mathrm{~V}$ for $1 \mathrm{~h}$, and then transferred onto a polyvinylidene fluoride (PVDF) membrane at $90 \mathrm{~V}$ for $90 \mathrm{~min}$ at $4^{\circ} \mathrm{C}$. Transfer buffer contained 10\% (v/v) $10 \times$ Tris/Glycine buffer (Bio-Rad, Laboratories), 15\% (v/v) methanol and $0.01 \%(\mathrm{w} / \mathrm{v})$ SDS. After $2 \mathrm{~h}$ incubation in chemiluminescent blocker (Millipore, Temecula, CA), the membranes were rinsed in TBST [10\% (v/v) $10 \times$ TBS buffer (BioRad, Laboratories), 0.1\% (v/v) Tween-20] and incubated with the primary antibodies against PEPT1 (1:500; Bioworld) and $\beta$-actin (1:3000; Bioworld) overnight at $4^{\circ} \mathrm{C}$. The blots were washed for $30 \mathrm{~min}$ in TBST and probed with the HRP-labeled secondary antibody (1:5000; Bioworld) for $1.5 \mathrm{~h}$ at room temperature and then washed again. Proteins were visualized using a chemical luminescence imaging analyzer (ImageQuan LAS 400; GE Healthcare Life Sciences, Pittsburgh, PA, USA) after being incubated with ECL western blotting substrate (Thermo Fisher Scientific Inc., Rockford, IL, USA). Band intensities were quantified using Quantity One software (Bio-Rad Laboratories). PEPT1 expression was normalized to $\beta$-actin.

\subsection{Proteome analysis}

\subsubsection{Sample preparation}

Proteins were isolated from epithelial cells of jejunal mucosa as above described, then re-suspended in $8 \mathrm{~mol} \cdot \mathrm{L}^{-1}$ urea and precipitated by adding three volumes of pre-cooled acetone. The samples were incubated at $-20^{\circ} \mathrm{C}$ for $12 \mathrm{~h}$ and then centrifuged at $14000 \mathrm{r} \cdot \mathrm{min}^{-1}$ for $15 \mathrm{~min}$. The pellet was washed twice with the same volume of pre-cooled acetone, centrifuged for $5 \mathrm{~min}$, resuspended in $8 \mathrm{~mol} \cdot \mathrm{L}^{-1}$ urea and then reduced with $20 \mathrm{mmol} \cdot \mathrm{L}^{-1} \mathrm{DTT}$ at $60^{\circ} \mathrm{C}$ for $1 \mathrm{~h}$. Proteins were then alkylated in the dark with $40 \mathrm{mmol} \cdot \mathrm{L}^{-1}$ iodoacetamide at $22^{\circ} \mathrm{C}$ for $30 \mathrm{~min}$. The alkylation reaction was quenched by $10 \mathrm{mmol} \cdot \mathrm{L}^{-1}$ DTT and samples were diluted to $2 \mathrm{~mol} \cdot \mathrm{L}^{-1}$ urea with $\mathrm{HPLC}$ grade water. Protein concentration was determined by a BCA protein assay kit (Pierce, Rockford, IL, USA). An appropriate amount of trypsin was mixed with the sample at an enzyme-to-substrate ratio of 1:100, and tryptic digestion was performed at $37^{\circ} \mathrm{C}$ for $18 \mathrm{~h}$ in $100 \mathrm{mmol} \cdot \mathrm{L}^{-1}$ triethylammonium bicarbonate ( $\mathrm{pH} 8$; Sigma-Aldrich). The digested products were desalted with ZipTip (Millipore, Temecula, CA, USA) and freeze-dried for LC-MS/ MS analysis.

\subsubsection{LC-MS/MS analysis}

Dried trypsin-digested products were dissolved in $12 \mu \mathrm{L}$ of $0.1 \%$ FA, and identified by a nano-LC system (Eksigentekspert $^{\mathrm{TM}}$ nanoLC 425; AB Sciex, Framingham, MA, USA) coupled to an AB Sciex Triple TOF ${ }^{\circledR} 6600$ system (AB Sciex). Peptides were trapped in a Chrom XP nanoLC C18 trap column ( $350 \mu \mathrm{m}$ id $\times 0.5 \mathrm{~mm}$ long, $3 \mu \mathrm{m}, 120 \AA)$, and eluted at a flow rate of $300 \mathrm{~nL} \cdot \mathrm{min}^{-1}$ into a reversephase C18 column ( $75 \mu \mathrm{m}$ id $\times 15 \mathrm{~cm}$ long, $3 \mu \mathrm{m}, 120 \AA)$. A linear gradient elution was applied using $3 \%$ to $36 \%$ acetonitrile in $0.1 \%$ formic acid with a total running time of $120 \mathrm{~min}$. Mass spectra were recorded in positive-ion and high-sensitivity mode with a resolution of 35000 full-width half-maximum. The nanospray needle voltage was $2300 \mathrm{~V}$. For collision induced dissociation tandem mass spectrometry (CID MS/MS), the mass window for precursor ion selection of the quadrupole mass analyzer was set to $\pm 2 \mathrm{~m} / \mathrm{z}$. The precursor ions were fragmented in a collision cell using nitrogen as the collision gas. Advanced information dependent acquisition (IDA) was used to obtain MS/MS spectra.

\subsubsection{Mass data analysis}

The MS generated files were searched against the UniProt, NCBI and common MS contaminant databases using Mascot 2.5 (Matrix Science, Boston, MA, USA) software. The tolerance for MS1 and MS2 error was set to $50 \mathrm{mg} \cdot \mathrm{kg}^{-1}$ and $50 \mathrm{Da}$, respectively. A maximum of 2 trypsin miss cleavages were allowed. Peptides were assumed to have a charge of $2+, 3+$ or $4+$. The mass input was assumed to be mono-isotopic mass. Decoy database was used to control the false discovery rate (FDR) less than $1 \%$. The search result was exported to CSV file for label-free quantification using in-house software.

Label-free quantification was performed by using normalized spectral index $\left(\mathrm{SI}_{n}\right)$ as previously described $^{[25,26]}$. $_{n}$ combines four MS abundance features: peptide count, spectral count, total ion intensities and protein length. This calculation eliminates variations between replicate MS measurements and permits quantitative reproducibility and highly significant quantification of the same and different samples ${ }^{[25]}$. The equations are: 


$$
\begin{gathered}
\mathrm{SI}=\sum_{k=1}^{\mathrm{pn}}\left(\sum_{j=1}^{\mathrm{sc}} i j\right) k \\
\mathrm{SI}_{\mathrm{GI}}=\mathrm{SI} / \sum_{j=1}^{n} \mathrm{SI}_{j} \\
\mathrm{SI}_{n}=\mathrm{SI}_{\mathrm{GI}} / L
\end{gathered}
$$

where $p n$ is the number of peptides identified for that protein, sc is the spectral count for the peptide $k, i$ is the fragment ion intensity of peptide $k, j$ is the $j$ th spectral count of sc total spectral counts for peptide $k$, GI is the global/total intensity.

Protein mapping was performed by matching at least two peptides to a designated protein that was annotated to a gene ID. To simplify the interpretation of the complicated nanoLC-MS/MS data, pairwise comparisons were performed between any one of the meat protein groups and the casein group. Only those proteins with $P<0.05$ were considered to be significantly different. Mascot search results are shown in the Table S2.

\subsubsection{GO and KEGG pathway analysis}

For interpretation of the functions of differentially expressed proteins, GO (gene ontology) and KEGG (Kyoto encyclopedia of genes and genomes) pathway analyses were performed by using the Enrichr program $^{[27]}$. $P<0.05$ in the Fisher exact test was considered for the identification of values that were significantly different.

\subsubsection{Gene set enrichment analysis}

Gene set enrichment analysis (GSEA) analysis was performed according to the procedure of Song et al. ${ }^{[28]}$. GSEA has multiple advantages over analysis of individual genes or proteins ${ }^{[29,30]}$. It is well accepted that the GSEA method can be applied to proteomic data, although it was initially developed for genomic data ${ }^{[29]}$. Thus, the protein set rather than the gene set was used in the present study. To interpret the GSEA results more intuitively, enrichment maps were generated with significant protein sets $(P<0.05$, FDR $<0.25)$ by using the Enrichment Map plugin v2.1.026 of Cytoscape v3.2.1 (the Institute of Systems Biology, Seattle, USA).

\subsection{Histological observations}

The formalin-fixed jejunal tissues were embedded in paraffin and cut transversely into $7 \mu \mathrm{m}$ thick sections by a microtome. The sections were transferred to a graded ethanol series and cleaned by xylene ${ }^{[11]}$. The sections were stained with hematoxylin and eosin. The images were captured by a light microscope (BH-2, Olympus, Tokyo,
Japan) and the morphological parameters were measured with Image Pro Plus software (Media Cybernetics, Silver Spring, MD, USA). Villus height was measured from the baseline to the tip of the highest one. The crypt depth was measured from the baseline to the bottom of the deepest one $^{[31]}$. Each tissue sample had two replicates, and ten visual fields were selected for further analysis.

\subsection{Statistical analysis}

The effect of diet on the variables was evaluated by oneway ANOVA and Duncan's multiple-range test with the program SAS 9.1.3 (SAS Institute Inc, Cary, NC, USA).

\section{Results}

\subsection{PEPT1 level}

The Pept $1 \mathrm{mRNA}$ levels in the jejunum were higher for the beef and chicken protein groups $(P<0.05$, Fig. 1$)$, compared to the casein group, while no significant difference was observed for the fish and pork protein groups $(P>0.05$, Fig. 1). Western blotting gave similar results at the PEPT1 protein level (Fig. 2). The relative PEPT1 abundance of the chicken protein group was the highest, and the lowest was observed for the pork protein group $(P<0.05$, Fig. $2 a)$. These results indicated that dietary protein did affect PEPT1 abundance in rat jejunum and may reflect the development of intestinal morphology.

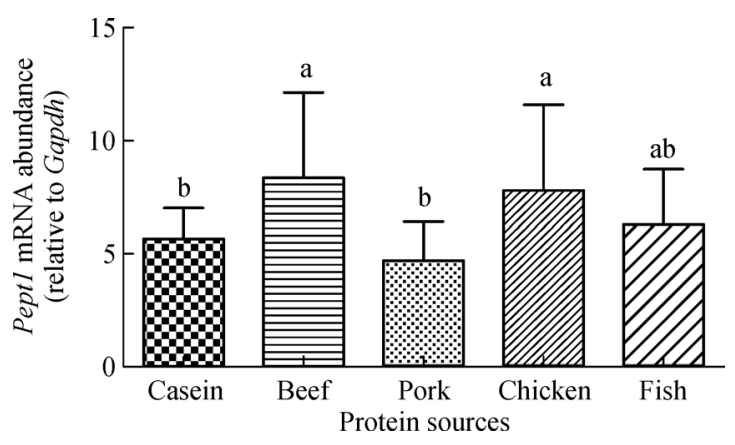

Fig. 1 PEPT1 mRNA levels in rat jejunum. Pept1 mRNA expression was quantified by qRT-PCR. Data are shown as means with standard deviations; there were 10 biological replicates in each group; values labeled a, b, differed significantly $(P<0.05)$.

\subsection{Histological parameters}

Villus height differed with dietary protein $(P<0.05$, Table 2). The chicken protein group had smaller villus height $(P<0.05)$, compared to the casein group, but no significant difference $(P>0.05)$ was observed between the beef, pork or fish protein groups and the casein group. Crypt depth did not differ $(P>0.05$, Table 2$)$ between any two protein groups. When data were combined, the ratio of 
(a)

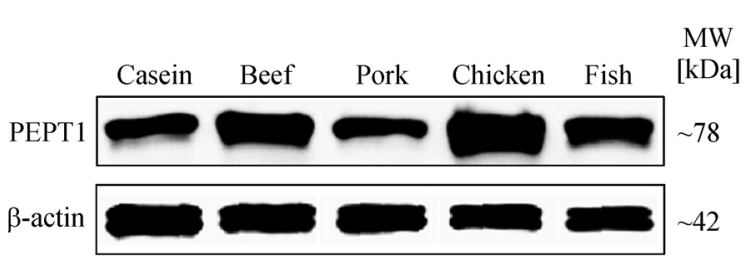

(b)

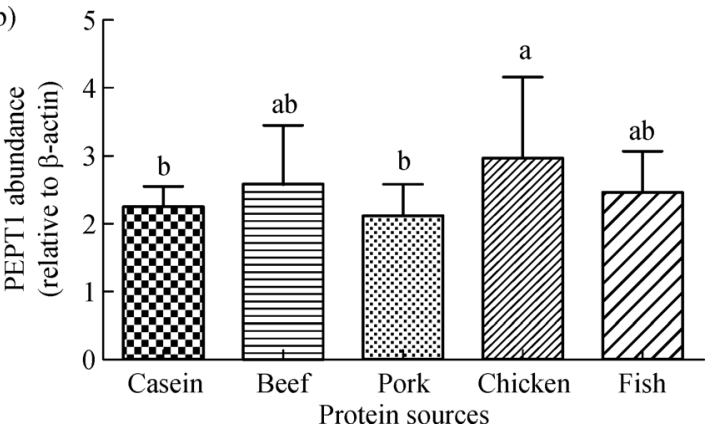

Fig. 2 PEPT1 protein levels in rat jejunum. (a) The PEPT1 band appeared at $\sim 78 \mathrm{kDa}$ and the control $\beta$-actin band appeared at $\sim 42 \mathrm{kDa}$; (b) the densities of PEPT1 bands were quantified and normalized to $\beta$-actin, to indicate the PEPT1 protein expression level. Data are shown as means and standard deviations, biological replicates $n=10$ for each group; values labeled a, b, differed significantly $(P<0.05)$.

villus height to crypt depth (V/C) was the greatest for the pork protein group, but the smallest for the chicken protein group $(P<0.05$, Table 2$)$.

\subsection{Differentially expressed proteins}

Label-free LC-MS/MS identified hundreds of unique proteins from all the jejunal mucosa (Table S2). As compared to the casein group, pork protein diet had the greatest number of differentially expressed proteins, followed by chicken, fish and beef protein groups $(102$, 66, 53 and 49, respectively, Table S3). Hierarchical clustering revealed the differences between pork protein group and any of the other three meat protein groups

Table 2 Histological measurements of the jejunal mucosa

\begin{tabular}{|c|c|c|c|}
\hline Dietary protein & Villus height $/ \mu \mathrm{m}$ & Crypt depth/ $\mu \mathrm{m}$ & $\mathrm{V} / \mathrm{C}$ ratio \\
\hline Casein & $528 \pm 53^{\mathrm{a}}$ & $176 \pm 11$ & $3.0 \pm 0.41^{\mathrm{ab}}$ \\
\hline Beef protein & $490 \pm 66^{\mathrm{ab}}$ & $171 \pm 11$ & $2.9 \pm 0.53^{\mathrm{ab}}$ \\
\hline Pork protein & $545 \pm 98^{\mathrm{a}}$ & $175 \pm 6$ & $3.1 \pm 0.52^{\mathrm{a}}$ \\
\hline Chicken protein & $450 \pm 22^{\mathrm{b}}$ & $179 \pm 18$ & $2.5 \pm 0.29^{b}$ \\
\hline Fish protein & $509 \pm 42^{\mathrm{ab}}$ & $181 \pm 25$ & $2.9 \pm 0.47^{\mathrm{ab}}$ \\
\hline
\end{tabular}

Note: Data are presented as mean $\pm \mathrm{SD}, n=10$ for each group; values with different superscript letters indicate significant difference $(P<0.05)$.

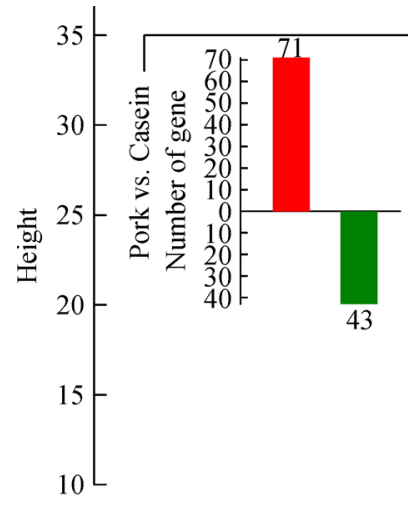

Upregulated

Downregulated
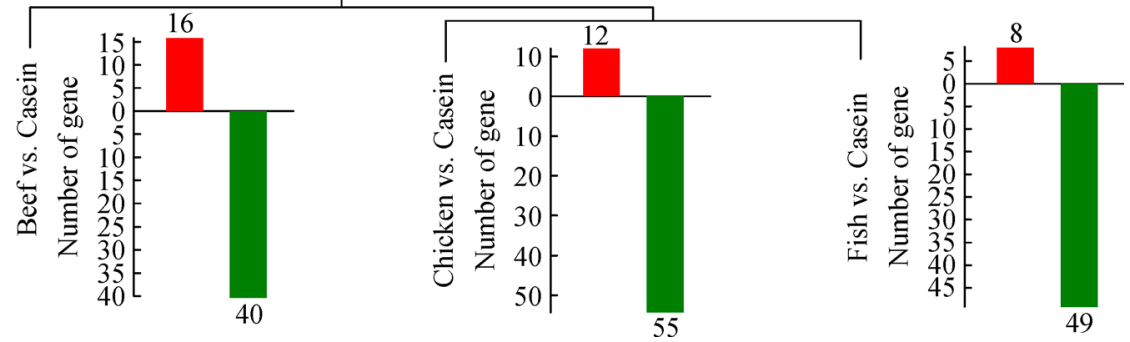

Fig. 3 Hierarchical clustering of differential membrane proteins. The four inserting coordinates show the number of upregulated (red) and downregulated (green) differential proteins. The length of vertical lines between any two coordinates indicates the differences between groups, the longer the lines were, the greater the differences were. 
(Fig. 3). As compared to the casein group, the number of upregulated proteins in the pork protein group was greater than that of downregulated proteins. However, intake of beef, chicken and fish led to downregulation of a greater number of proteins.

\subsection{GO and KEGG analyses}

The RT-PCR, western blotting and histological results revealed significant differences in PEPT1 level and V/C ratio between chicken and pork protein groups. To obtain information about the underlying mechanism, GO and KEGG pathway analyses were performed using 66 differentially expressed proteins in the chicken protein group and 102 proteins in the pork protein group. As compared to the casein group, the chicken protein group showed significant differences in biological process, cellular component and molecular function (Tables 3-5). However, no significant differences were observed between the pork protein group and the casein group (data not shown).

In biological process categories, the enriched GO terms derived from upregulated proteins were related to cell junction organization, Fc-gamma receptor signaling pathway regulation of proton transport and oxidative phosphorylation $(P<0.05$, Table 3$)$. The terms derived from downregulated proteins were relevant to metabolic processes related to organic acids (e.g., uronic acid), protein polymerization and folding, digestion, and terpenoid and GTP metabolic process $(P<0.05$, Table 3$)$.

In cellular component categories, the terms derived from upregulated proteins were involved in the mitochondrial respiratory chain and cytochrome complex $(P<0.05$, Table 4). The terms derived from downregulated proteins were associated with myosin complex, brush border, NADH dehydrogenase and respiratory chain complex $(P<0.05$, Table 4$)$.

In molecular function categories, the terms derived from upregulated proteins were related to oxidoreductase activity, ubiquinol cytochrome-c reductase activity and amide transmembrane transporter activity $(P<0.05$, Table 5). The downregulated proteins were associated with organic acid binding, drug binding, electron carrier activity, fatty acid transporter activity, calcium-transporting ATPase and dipeptidyl peptidase activity $(P<0.05$, Table 5).

Table 3 Enriched GO terms in biological process categories for differential proteins

\begin{tabular}{|c|c|c|}
\hline Biological process _ GO term (GO ID) & Overlap & $P$-value \\
\hline \multicolumn{3}{|l|}{ Chicken protein vs. Casein _ upregulated } \\
\hline Cell junction organization (GO:0034330) & $2 / 189$ & 0.011 \\
\hline Immune response-regulating cell surface receptor signaling pathway involved in phagocytosis (GO:0002433) & $2 / 203$ & 0.012 \\
\hline Fc-gamma receptor signaling pathway involved in phagocytosis (GO:0038096) & $2 / 203$ & 0.012 \\
\hline Fc-gamma receptor signaling pathway (GO:0038094) & $2 / 203$ & 0.012 \\
\hline Fc receptor mediated stimulatory signaling pathway (GO:0002431) & $2 / 204$ & 0.012 \\
\hline Regulation of proton transport (GO:0010155) & $1 / 14$ & 0.013 \\
\hline Oxidative phosphorylation (GO:0006119) & $1 / 14$ & 0.013 \\
\hline \multicolumn{3}{|l|}{ Chicken protein vs. Casein _ downregulated } \\
\hline Cellular glucuronidation (GO:0052695) & $4 / 17$ & $<0.0001$ \\
\hline Uronic acid metabolic process (GO:0006063) & $4 / 18$ & $<0.0001$ \\
\hline Glucuronate metabolic process (GO:0019585) & $4 / 18$ & $<0.0001$ \\
\hline Flavonoid metabolic process (GO:0009812) & $3 / 11$ & $<0.0001$ \\
\hline Monocarboxylic acid metabolic process (GO:0032787) & $9 / 473$ & $<0.0001$ \\
\hline Metinoic acid metabolic process (GO:0042573) & $3 / 21$ & $<0.0001$ \\
\hline Protein polymerization (GO:0051258) & $4 / 64$ & $<0.0001$ \\
\hline Cellular protein complex assembly (GO:0043623) & $6 / 256$ & 0.0003 \\
\hline 'De novo' posttranslational protein folding (GO:0051084) & $3 / 49$ & 0.0007 \\
\hline 'De novo' protein folding (GO:0006458) & $3 / 54$ & 0.001 \\
\hline Diterpenoid metabolic process (GO:0016101) & $4 / 87$ & 0.0003 \\
\hline Terpenoid metabolic process (GO:0006721) & $4 / 97$ & 0.0004 \\
\hline GTP metabolic process (GO:0046039) & $5 / 258$ & 0.002 \\
\hline GTP catabolic process (GO:0006184) & $5 / 243$ & 0.002 \\
\hline Digestion (GO:0007586) & $3 / 66$ & 0.002 \\
\hline
\end{tabular}

Note: Overlap represents the numbers of identified proteins matching with GO terms. 
Table 4 Enriched GO terms in cellular component categories for differential proteins

\begin{tabular}{llc}
\hline Cellular component_GO term (GO ID) & Overlap & $P$-value \\
\hline Chicken protein vs. Casein_ upregulated & $1 / 8$ & 0.008 \\
Mitochondrial respiratory chain complex III (GO:0005750) & $1 / 8$ & 0.008 \\
Respiratory chain complex III (GO:0045275) & $1 / 14$ & 0.014 \\
Mitochondrial respiratory chain (GO:0005746) & $1 / 18$ & 0.017 \\
Cytochrome complex (GO:0070069) & & $</ 64$ \\
Chicken protein vs. Casein_downregulated & $3 / 25$ & 0.0001 \\
Myosin complex (GO:0016459) & $2 / 18$ & 0.002 \\
Myosin II complex (GO:0016460) & $3 / 40$ \\
Muscle myosin complex (GO:0005859) & $2 / 24$ \\
Mitochondrial nucleoid (GO:0042645) & $2 / 47$ & 0.0005 \\
Brush border (GO:0005903) & $2 / 47$ & 0.003 \\
NADH dehydrogenase complex (GO:0030964) & 0.013 \\
Respiratory chain complex I (GO:0045271) & & 0.0123 \\
\hline
\end{tabular}

Note: Overlap represents the numbers of identified proteins matching with GO terms.

Table 5 Enriched GO terms in molecular function categories for differential proteins

\begin{tabular}{|c|c|c|}
\hline Molecular function_GO term (GO ID) & Overlap & $P$-value \\
\hline \multicolumn{3}{|l|}{ Chicken protein vs. Casein _ upregulated } \\
\hline Oxidoreductase activity, acting on diphenols and related substances as donors, cytochrome as acceptor (GO:0016681) & $1 / 8$ & 0.008 \\
\hline Oxidoreductase activity, acting on diphenols and related substances as donors (GO:0016679) & $1 / 9$ & 0.009 \\
\hline Ubiquinol-cytochrome-c reductase activity (GO:0008121) & $1 / 8$ & 0.008 \\
\hline Amide transmembrane transporter activity (GO:0042887) & $1 / 10$ & 0.010 \\
\hline Oxidoreductase activity, acting on the aldehyde or oxo group of donors, NAD or NADP as acceptor (GO:0016620) & $1 / 31$ & 0.030 \\
\hline Oxidoreductase activity, acting on the aldehyde or oxo group of donors (GO:0016903) & $1 / 42$ & 0.039 \\
\hline \multicolumn{3}{|l|}{ Chicken protein vs. Casein _ downregulated } \\
\hline Monocarboxylic acid binding (GO:0033293) & $6 / 59$ & $<0.0001$ \\
\hline Isoprenoid binding (GO:0019840) & $5 / 38$ & $<0.0001$ \\
\hline Retinoid binding (GO:0005501) & $5 / 38$ & $<0.0001$ \\
\hline Retinoic acid binding (GO:0001972) & $4 / 23$ & $<0.0001$ \\
\hline Carboxylic acid binding (GO:0031406) & $6 / 200$ & 0.0001 \\
\hline Organic acid binding (GO:0043177) & $6 / 201$ & 0.0001 \\
\hline Fatty acid binding (GO:0005504) & $3 / 26$ & 0.0002 \\
\hline Drug binding (GO:0008144) & $4 / 93$ & 0.0005 \\
\hline Voltage-gated anion channel activity (GO:0008308) & $3 / 21$ & 0.0001 \\
\hline Wide pore channel activity (GO:0022829) & $3 / 23$ & 0.0001 \\
\hline Anion channel activity (GO:0005253) & $3 / 80$ & 0.004 \\
\hline Voltage-gated ion channel activity (GO:0005244) & $3 / 189$ & 0.037 \\
\hline Voltage-gated channel activity (GO:0022832) & $3 / 189$ & 0.037 \\
\hline Electron carrier activity (GO:0009055) & $4 / 107$ & 0.0008 \\
\hline Calcium-transporting ATPase activity (GO:0005388) & $1 / 9$ & 0.038 \\
\hline Fatty acid transporter activity (GO:0015245) & $1 / 9$ & 0.038 \\
\hline Dipeptidyl-peptidase activity (GO:0008239) & $1 / 11$ & 0.045 \\
\hline
\end{tabular}

Note: Overlap represents the numbers of identified proteins matching with GO terms. 
The enriched KEGG pathways derived from upregulated proteins were related to adherens junctions, focal adhesion, hippo signaling pathway, apoptosis, collecting duct acid secretion and galactose metabolism $(P<0.05$, Table 6). The downregulated proteins were related to glycolysis, retinol metabolism, ascorbate and aldarate metabolism, xenobiotics and drug metabolism.

Table 6 Enriched KEGG pathway for differential proteins

\begin{tabular}{lcc}
\hline KEGG Term & Overlap & $P$-value \\
\hline Chicken protein vs. Casein _ upregulated & & \\
Adherens junctions & $2 / 74$ & 0.004 \\
Focal adhesion & $2 / 202$ & 0.026 \\
Hippo signaling pathway & $2 / 153$ & 0.016 \\
Apoptosis & $2 / 140$ & 0.013 \\
Collecting duct acid secretion & $1 / 27$ & 0.035 \\
Galactose metabolism & $1 / 30$ & 0.039 \\
Chicken protein vs. Casein _downregulated & & \\
Starch and sucrose metabolism & $6 / 56$ & $2.24 \times 10^{-6}$ \\
Pentose and glucuronate interconversions & $5 / 36$ & $5.30 \times 10^{-6}$ \\
Fructose and mannose metabolism & $2 / 32$ & 0.02 \\
Ascorbate and aldarate metabolism & $5 / 27$ & $1.49 \times 10^{-6}$ \\
Retinol metabolism & $5 / 65$ & $7.47 \times 10^{-5}$ \\
Drug metabolism- other enzymes & $5 / 46$ & $1.59 \times 10^{-5}$ \\
Drug metabolism- cytochrome P450 & $5 / 69$ & $9.76 \times 10^{-5}$ \\
Metabolism of xenobiotics by cytochrome & $5 / 73$ & 0.0001 \\
P450 & & \\
\hline
\end{tabular}

Note: Overlap represents the numbers of identified proteins matching with KEGG terms.

\subsection{GSEA analysis}

GSEA analysis was performed in order to analyze biological functions at the level of protein sets instead of individual proteins. There were 4, 8, 10 and 5 protein sets, respectively in the beef, pork, chicken and fish protein groups that differed from those in the casein group (Table S4). The clustering and enrichment maps revealed that intake of four meat proteins regulated carbohydrate metabolism, biological oxidation, biological development and several signaling pathways (Figs. 4-5).

When compared to the casein group, the cluster of protein sets related to carbohydrate metabolism was significantly changed by intake of pork protein, which is reflected by increased glucose metabolism, glycogen storage, and starch and sucrose metabolism. Intake of chicken increased glucose metabolism, but inhibited starch and sucrose metabolism. Ingestion of fish protein inhibited pentose and glucoronate interconversions, and ascorbate and aldarate metabolism. Beef protein showed a high similarity to casein.

For the protein sets related to biological oxidation, as compared to control (casein), intake of chicken protein increased NFE2L2, drug metabolism and metabolism of xenobiotics by cytochrome P450, but decreased oxidative phosphorylation. However, intake of pork protein inhibited respiratory electron transport. Intake of beef protein also inhibited respiratory electron transport, but increased NFE2L2. Fish protein intake did not affect any protein sets related to biological oxidation.

In addition, intake of chicken protein inhibited the protein sets related to gap junction, developmental biology and axon guidance. Intake of fish protein did not inhibit the protein sets related to gap junction, but inhibited cGMPPKG signaling pathway and GPCR downstream signaling pathway.

\section{Discussion}

The jejunum is the main site for nutrient absorption/ transport and its development affects bioavailability of food nutrients ${ }^{[32]}$. Villus height and crypt depth may reflect the development of the small intestine. Greater V/C ratio indicates higher capacity of nutrient absorption and transport from the intestinal lumen to the bloodstream ${ }^{[10,11]}$. During maturation, the expression of proteins related to brush border assembly, metabolism of glucose, amino acids, and fatty acids increases in intestinal epithelial cells along the crypt-villus axis ${ }^{[33,34]}$. PEPT1 is located in the brush border membrane of enterocytes and is responsible for the transport of dipeptides and tripeptides $^{[8,9]}$. A 7-day feeding study found lower $\mathrm{V} / \mathrm{C}$ ratio but higher abundance of PEPT1 in the jejunum for the rats fed beef protein than those of the rats fed pork, chicken and fish proteins ${ }^{[20]}$. In the present study, the chicken protein group had higher levels of PEPT1 mRNA and protein, but lower V/C ratio than the pork protein group. Compared to casein, intake of chicken protein had a stronger influence on PEPT1 level and morphology of rat jejunum than in the pork protein group. This could be because the products of protein digestion are likely to be the metabolic signals triggering the modulation of PEPT1 expression ${ }^{[35]}$, and proliferation and differentiation of the intestinal epithelial cells $^{[36]}$. In addition, the gastric leptin may selectively mediate peptide transport across the intestinal epithelium $^{[37]}$.

Diet has a broad influence on the small intestine. For example, protein limitation in diet can upregulate the proteins involved in protein/carbohydrate digestion, intestinal mucosal tight junction and cell adhesion, and the immune response to foreign antigens in the jejunal mucosa of pigs, but downregulate amino acid transport, innate and auto immunity, as well as cell proliferation and apopto$\operatorname{sis}^{[38]}$. In the present study, GO and KEGG pathway analyses indicated that, under the normal dose, intake of chicken protein induced upregulation of respiratory chain, oxidative phosphorylation and oxidoreductase activity, cell 


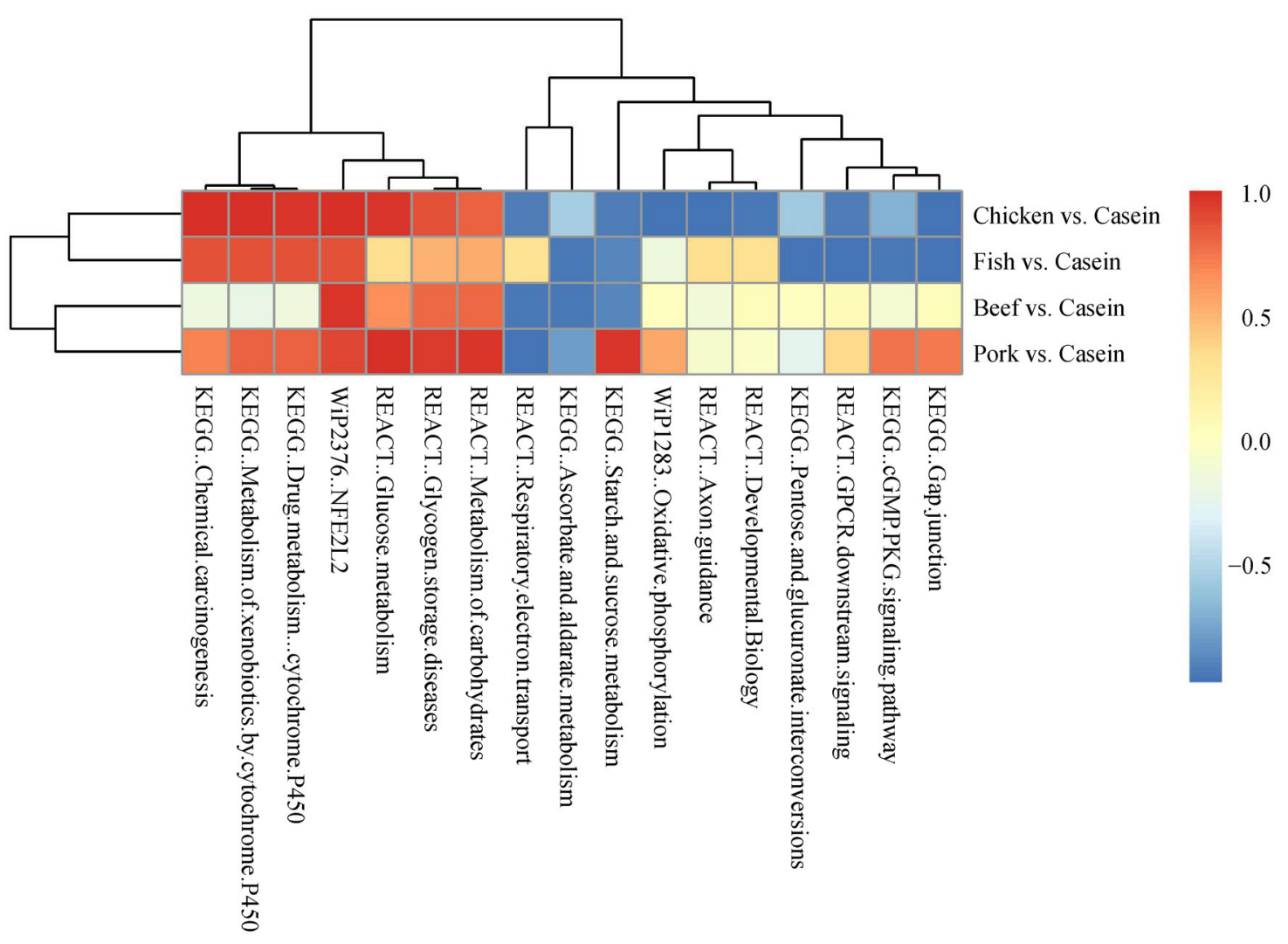

Fig. 4 Clustered heat map of jejunal membrane protein sets. The rows are the clusters of differential protein sets (pathways) responding to one meat protein diet as compared to the casein group, and the columns are individual protein sets (pathways). The color scale represents the fraction of regulated protein sets; the more intense the color, the higher the fraction. Red means enriched, while blue means suppressed as compared to casein.
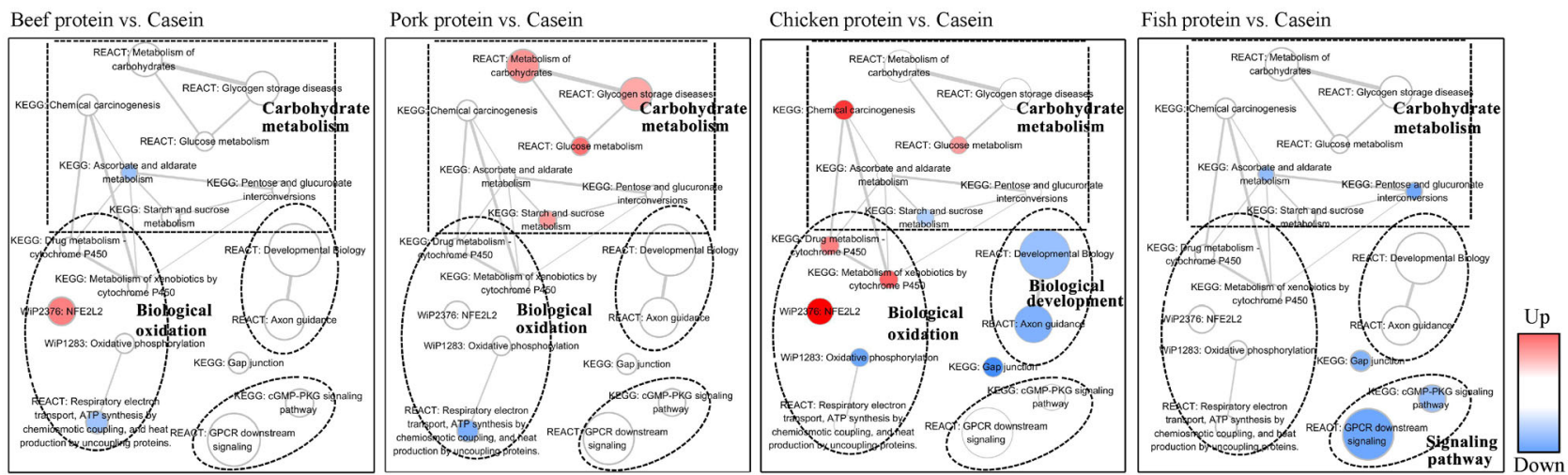

Fig. 5 Network of protein sets for different protein groups enriched by GSEA analysis. The network was produced using Cytoscape. Nodes represent the enriched protein sets. The red nodes represent upregulated protein sets and the blue nodes represent downregulated protein sets. The sizes of nodes are proportional to the total number of proteins within each set (from 15 to 500). The lines between nodes represent the overlap score (Jaccard and overlap coefficient $>0.375$ ) depending on the number of proteins that two protein sets share.

junction organization, and regulation of proton transport. This is closely linked to the process of biologic oxidization $^{[39]}$, accompanied by energy release to meet immediate energy needs of all the biological reactions ${ }^{[40]}$. This process would further regulate amide transmembrane transporter activity, glucose and oligopeptide transporter expression, galactose metabolism, and consequently the development of intestinal morphology. Glucose-mediated 
energy homeostasis is an upstream event involved in regulation of the Hippo signaling pathway ${ }^{[41]}$, which can regulate cell proliferation and apoptosis of organs. Consequently, intake of different meat proteins could change jejunal absorption capacity by regulating intestinal morphology.

In addition, dietary proteins also regulated intercellular substance transport and signal transduction (cell junction organization, adherens junctions, and focal adhesion) and then collecting duct acid secretion via neural control. This can be attributed to the differences in amino acid composition of different meats. For example, L-glutamine can regulate the expression of intestinal tight junction protein in weanling piglets ${ }^{[42]}$. Dietary $\mathrm{N}$ reduction led to the downregulation of cadherin $17^{[43]}$. Intake of chicken protein can increase focal adhesion, prevent insulin resistance, and consequently increase glucose metabo$\operatorname{lism}^{[44]}$.

Also, intake of chicken protein reduced carbohydrate metabolism, ascorbate and metabolism of aldarate, retinol, and xenobiotics. These processes can be realized by inhibiting changes in regulating respiratory chain complexes, anion channels, and transporter activity. Peptides derived from dietary chicken protein might reduce changes in regulating calcium-transporting ATPase activity to inhibit calcium transport, dipeptidyl peptidase activity to promote dipeptide transport, and fatty acid transporter activity to inhibit fatty acid transport.

\section{Conclusions}

The types of dietary proteins had a significant effect on PEPT1 expression and proteome profile of rat jejunum. When compared to casein, intake of chicken protein had the strongest effect on PEPT1 expression level and V/C ratio of the jejunum, but the weakest for the pork protein group. Intake of chicken protein increased oligopeptide transport by increasing PEPT1 expression level, downregulating biological processes involving energy production and reducing dipeptidyl peptidase activity. Also, it decreased oligopeptide absorption capacity by inducing the Hippo signaling pathway related to intestinal morphology. These findings provide new insights into protein nutrition.

Supplementary materials The online version of this article at https://doi. org/10.15302/J-FASE-2018206 contains supplementary materials (Tables S1-S4).

Acknowledgements This work was supported by the National Natural Science Foundation of China (31471600, 31530054).

Compliance with ethics guidelines Mengjie Li, Chunbao Li, Shangxin Song, Xinglian Xu, and Guanghong Zhou declare that they have no conflicts of interest or financial conflicts to disclose.

All applicable institutional and national guidelines for the care and use of animals were followed.

\section{References}

1. Drozdowski L, Thomson A B R. Intestinal mucosal adaptation. World Journal of Gastroenterology, 2006, 12(29): 4614-4627

2. Kuhla S, Rudolph P E, Albrecht D, Schoenhusen U, Zitnan R, Tomek W, Huber K, Voigt J, Metges C C. A milk diet partly containing soy protein does not change growth but regulates jejunal proteins in young goats. Journal of Dairy Science, 2007, 90(9): 4334-4345

3. Tamada H, Nezu R, Matsuo Y, Imamura I, Takagi Y, Okada A. Alanyl glutamine-enriched total parenteral nutrition restores intestinal adaptation after either proximal or distal massive resection in rats. Journal of Parenteral and Enteral Nutrition, 1993, 17(3): 236242

4. Sukhotnik I, Mogilner J G, Lerner A, Coran A G, Lurie M, Miselevich I, Shiloni E. Parenteral arginine impairs intestinal adaptation following massive small bowel resection in a rat model. Pediatric Surgery International, 2005, 21(6): 460-465

5. Tossou M C B, Liu H, Bai M, Chen S, Cai Y, Duraipandiyan V, Liu $\mathrm{H}$, Adebowale T O, Al-Dhabi N A, Long L, Tarique H, Oso A O, Liu G, Yin Y. Effect of high dietary tryptophan on intestinal morphology and tight junction protein of weaned pig. BioMed Research International, 2016, Article ID 2912418

6. Sun Y, Wu Z, Li W, Zhang C, Sun K, Ji Y, Wang B, Jiao N, He B, Wang W, Dai Z, Wu G. Dietary L-leucine supplementation enhances intestinal development in suckling piglets. Amino Acids, 2015, 47(8): 1517-1525

7. Daniel H, Kottra G. The proton oligopeptide cotransporter family SLC15 in physiology and pharmacology. Pflügers Archiv, 2004, 447 (5): 610-618

8. Fei Y J, Ganapathy V, Leibach F H. Molecular and structural features of the proton-coupled oligopeptide transporter superfamily. Progress in Nucleic Acid Research and Molecular Biology, 1998, 58: 239-261

9. Ganapathy L F H, Leibach F H. Is intestinal peptide transport energized by a proton gradient? American Journal of Physiology, 1985, 249(2): G153-G160

10. Alpers D H. Protein synthesis in intestinal mucosa: the effect of route of administration of precursor amino acids. Journal of Clinical Investigation, 1972, 51(1): 167-173

11. Rajkovic V, Djolai M, Matavulj M. Alterations in jejunal morphology and serotonin-containing enteroendocrine cells in peripubertal male rats associated with subchronic atrazine exposure. Ecotoxicology and Environmental Safety, 2011, 74(8): 2304-2309

12. Hopkins A L, Groom C R. The druggable genome. Nature Reviews: Drug Discovery, 2002, 1(9): 727-730

13. Madani S, Prost J, Belleville J. Dietary protein level and origin (casein and highly purified soybean protein) affect hepatic storage, plasma lipid transport, and antioxidative defense status in the rat. Nutrition, 2000, 16(5): 368-375

14. Nässl A M, Rubio-Aliaga I, Sailer M, Daniel H. The intestinal peptide transporter PEPT1 is involved in food intake regulation in mice fed a high-protein diet. PLoS One, 2011, 6(10): e26407

15. Shiraga T, Miyamoto K, Tanaka H, Yamamoto H, Taketani Y, Morita K, Tamai I, Tsuji A, Takeda E. Cellular and molecular mechanisms of dietary regulation on rat intestinal $\mathrm{H}^{+}$/Peptide 
transporter PepT1. Gastroenterology, 1999, 116(2): 354-362

16. Gilbert J A, Bendsen N T, Tremblay A, Astrup A. Effect of proteins from different sources on body composition. Nutrition, Metabolism, and Cardiovascular Diseases, 2011, 21(S2): B16-B31

17. Pereira P M, Vicente A F. Meat nutritional composition and nutritive role in the human diet. Meat Science, 2013, 93(3): 586-592

18. Wen S, Zhou G, Song S, Xu X, Voglmeir J, Liu L, Zhao F, Li M, Li $\mathrm{L}, \mathrm{Yu} \mathrm{X}, \mathrm{Bai} \mathrm{Y}, \mathrm{Li}$ C. Discrimination of in vitro and in vivo digestion products of meat proteins from pork, beef, chicken, and fish. Proteomics, 2015, 15(21): 3688-3698

19. Herrera-Ruiz D, Knipp G T. Current perspectives on established and putative mammalian oligopeptide transporters. Journal of Pharmacological Sciences, 2003, 92(4): 691-714

20. Li M, Li C, Song S, Zhao F, Xu X, Zhou G. Meat proteins had different effects on oligopeptide transporter PEPT1 in the small intestine of young rats. International Journal of Food Sciences and Nutrition, 2016, 67(8): 995-1004

21. Reeves P G, Nielsen F H, Fahey G C Jr. AIN-93 purified diets for laboratory rodents: final report of the American Institute of Nutrition ad hoc writing committee on the reformulation of the AIN-76A rodent diet. Journal of Nutrition, 1993, 123(11): 1939-1951

22. Zhu Y, Lin X, Zhao F, Shi X, Li H, Li Y, Zhu W, Xu X, Li C, Zhou G. Meat, dairy and plant proteins alter bacterial composition of rat gut bacteria. Scientific Reports, 2015, 5(1): 15220

23. Song S, Hooiveld G J, Li M, Zhao F, Zhang W, Xu X, Muller M, Li C, Zhou G. Dietary soy and meat proteins induce distinct physiological and gene expression changes in rats. Scientific Reports, 2016, 6(1): 20036

24. Livak K J, Schmittgen T D. Analysis of relative gene expression data using real-time quantitative PCR and the $2^{-\Delta \Delta C_{\mathrm{T}}}$ method. Methods, 2001, 25(4): 402-408

25. Griffin N M, Yu J, Long F, Oh P, Shore S, Li Y, Koziol J A, Schnitzer J E. Label-free, normalized quantification of complex mass spectrometry data for proteomic analysis. Nature Biotechnology, 2010, 28(1): 83-89

26. Xu B, Zhang Y, Zhao Z, Yoshida Y, Magdeldin S, Fujinaka H, Ismail T A, Yaoita E, Yamamoto T. Usage of electrostatic eliminator reduces human keratin contamination significantly in gel-based proteomics analysis. Journal of Proteomics, 2011, 74(7): 10221029

27. Chen E Y, Tan C M, Kou Y, Duan Q, Wang Z, Meirelles G V, Clark N R, Ma'ayan A. Enrichr: interactive and collaborative HTML5 gene list enrichment analysis tool. BMC Bioinformatics, 2013, 14 (1): 128

28. Song S, Hooiveld G J, Zhang W, Li M, Zhao F, Zhu J, Xu X, Muller $\mathrm{M}, \mathrm{Li} \mathrm{C}$, Zhou G. Comparative proteomics provides insights into metabolic responses in rat liver to isolated soy and meat proteins. Journal of Proteome Research, 2016, 15(4): 1135-1142

29. Subramanian A, Tamayo P, Mootha V K, Mukherjee S, Ebert B L, Gillette M A, Paulovich A, Pomeroy S L, Golub T R, Lander E S, Mesirov J P. Gene set enrichment analysis: a knowledge-based approach for interpreting genome-wide expression profiles. Proceedings of the National Academy of Sciences of the United States of America, 2005, 102(43): 15545-15550
30. Abatangelo L, Maglietta R, Distaso A, D'Addabbo A, Creanza T M, Mukherjee S, Ancona N. Comparative study of gene set enrichment methods. BMC Bioinformatics, 2009, 10(1): 275

31. Carneiro-Filho B A, Oriá R B, Wood Rea K, Brito G A, Fujii J, Obrig T, Lima A A, Guerrant R L. Alanyl-glutamine hastens morphologic recovery from 5-fluorouracil-induced mucositis in mice. Nutrition, 2004, 20(10): 934-941

32. Jahan-Mihan A, Luhovyy B L, El Khoury D, Anderson G H. Dietary proteins as determinants of metabolic and physiologic functions of the gastrointestinal tract. Nutrients, 2011, 3(5): 574603

33. Yang H, Wang X, Xiong X, Yin Y. Energy metabolism in intestinal epithelial cells during maturation along the crypt-villus axis. Scientific Reports, 2016, 6(1): 31917

34. Chang J, Chance M R, Nicholas C, Ahmed N, Guilmeau S, Flandez M, Wang D, Byun D S, Nasser S, Albanese J M, Corner G A, Heerdt B G, Wilson A J, Augenlicht L H, Mariadason J M. Proteomic changes during intestinal cell maturation in vivo. Journal of Proteomics, 2008, 71(5): 530-546

35. Spanier B. Transcriptional and functional regulation of the intestinal peptide transporter PEPT1. Journal of Physiology, 2014, 592(5): 871-879

36. Ayyadurai S, Charania M A, Xiao B, Viennois E, Zhang Y, Merlin D. Colonic miRNA expression/secretion, regulated by intestinal epithelial PepT1, plays an important role in cell-to-cell communication during colitis. PLoS One, 2014, 9(2): e87614

37. Buyse M, Berlioz F, Guilmeau S, Tsocas A, Voisin T, Péranzi G, Merlin D, Laburthe M, Lewin M J, Rozé C, Bado A. PepT1mediated epithelial transport of dipeptides and cephalexin is enhanced by luminal leptin in the small intestine. Journal of Clinical Investigation, 2001, 108(10): 1483-1494

38. Qin C, Qiu K, Sun W, Jiao N, Zhang X, Che L, Zhao H, Shen H, Yin J. A proteomic adaptation of small intestinal mucosa in response to dietary protein limitation. Scientific Reports, 2016, 6(1): 36888

39. Papa S, Martino P L, Capitanio G, Gaballo A, De Rasmo D, Signorile A, Petruzzella V. The oxidative phosphorylation system in mammalian mitochondria. Advances in Experimental Medicine and Biology, 2012, 942: 3-37

40. Senior A E. ATP synthesis by oxidative phosphorylation. Physiological Reviews, 1988, 68(1): 177-231

41. Wang W, Xiao Z D, Li X, Aziz K E, Gan B, Johnson R L, Chen J. AMPK modulates Hippo pathway activity to regulate energy homeostasis. Nature Cell Biology, 2015, 17(4): 490-499

42. Wang H, Zhang C, Wu G, Sun Y, Wang B, He B, Dai Z, Wu Z. Glutamine enhances tight junction protein expression and modulates corticotropin-releasing factor signaling in the jejunum of weanling piglets. Journal of Nutrition, 2015, 145(1): 25-31

43. Elfers K, Marr I, Wilkens M R, Breves G, Langeheine M, Brehm R, Muscher-Banse A S. Expression of tight junction proteins and cadherin 17 in the small intestine of young goats offered a reduced $\mathrm{N}$ and/or Ca diet. PLoS One, 2016, 11(4): e0154311

44. Bisht B, Srinivasan K, Dey C S. In vivo inhibition of focal adhesion kinase causes insulin resistance. Journal of Physiology, 2008, 586 (16): $3825-3837$ 